\title{
Magnetic resonance-guided laser interstitial thermal therapy: report of a series of pediatric brain tumors
}

\author{
Zulma Tovar-Spinoza, MD, and Hoon Choi, MD \\ Department of Neurosurgery, SUNY Upstate Medical University, Syracuse, New York
}

\begin{abstract}
OBJECTIVE Magnetic resonance-guided laser interstitial thermal therapy (MRgLITT) is a novel, minimally invasive treatment that has multiple advantages in pediatric use and broad applicability for different types of lesions. Here, the authors report the preliminary results of the first series of pediatric brain tumors treated with MRgLITT at Golisano Children's Hospital in Syracuse, New York.
\end{abstract}

METHODS Pediatric brain tumors treated with MRgLITT between February 2012 and August 2014 at Golisano Children's Hospital were evaluated retrospectively. Medical records, radiological findings, surgical data, complications, and results of tumor volumetric analyses were reviewed. The Visualase thermal laser system (Medtronic) was used in all MRgLITT procedures.

RESULTS This series included 11 patients with 12 tumors (pilocytic astrocytoma, ependymoma, medulloblastoma, choroid plexus xanthogranuloma, subependymal giant cell astrocytoma, and ganglioglioma). A single laser and multiple overlapping ablations were used for all procedures. The mean laser dose was $10.23 \mathrm{~W}$, and the mean total ablation time was 68.95 seconds. The mean initial target volume was $6.79 \mathrm{~cm}^{3}$, and the mean immediate post-ablation volume was $7.86 \mathrm{~cm}^{3}$. The mean hospital stay was 3.25 days, and the mean follow-up time was 24.5 months. Tumor volume decreased in the first 3 months after surgery $(n=11 ; p=0.007)$ and continued to decrease by the 4 - to 6 -month followup $\left(n=11 ;\right.$ mean volume $\left.2.61 \mathrm{~cm}^{3} ; p=0.009\right)$. Two patients experienced post-ablation complications: transient right leg weakness in one patient, and transient hemiparesis, akinetic mutism, and eye movement disorder in the other.

CONCLUSIONS Magnetic resonance-guided laser interstitial thermal therapy is an effective first- or second-line treatment for select pediatric brain tumors. Larger multiinstitutional clinical trials are necessary to evaluate its use for different types of lesions to further standardize practices.

http://thejns.org/doi/abs/10.3171/2015.11.PEDS15242

KEY WORDS laser interstitial thermal therapy; laser; ablation; pediatric; brain tumor; minimally invasive surgery; oncology

$\mathrm{M}$ AGNETIC resonance-guided laser interstitial thermal therapy (MRgLITT) ${ }^{5,8,9}$ has assumed an important role as a percutaneous minimally invasive alternative to conventional surgical interventions for brain tumors in adults; $;^{2-5,10,12,16,20,21,24,26-28,33}$ however, publications describing its use in pediatric patients are scarce., ${ }^{6,13,25} \mathrm{La}-$ ser interstitial thermal therapy is accomplished by using a high-power laser placed inside the tumor to deliver therapy. ${ }^{17,18,24}$ It is hypothesized that this procedure achieves effective tumor destruction ${ }^{41}$ and provides advantages such as decreased discomfort, reduced overall morbidity, and fewer complications compared with conventional surgical techniques. ${ }^{20}$
We previously described the MRgLITT technology and its use in children. ${ }^{6,25,34}$ Here, we report the preliminary results of the first series of heterogeneous pediatric brain tumors treated with MRgLITT at Golisano Children's Hospital in Syracuse, New York.

\section{Methods \\ Study Design}

Approval from the SUNY Upstate Medical University Institutional Review Board was obtained to retrospectively and prospectively study pediatric patients with diagnosed brain tumors treated with MRgLITT between February

ABBREVIATIONS MRgLITT = MR-guided laser interstitial thermal therapy; NF1 = neurofibromatosis Type 1; SEGA = subependymal giant cell astrocytoma . SUBMITTED April 19, 2015. ACCEPTED November 2, 2015. 
2012 and August 2014. Medical records, radiological findings, surgical data, complications, and results of tumor volumetric analyses were reviewed.

\section{Participants}

Patients were selected based on clinical presentation, tumor location, tumor progression, and/or palliative treatment in cases refractory to oncological treatment (Table 1). This series included 12 tumors in 11 patients ( 6 boys, 5 girls) whose mean age was 10.3 years (range $4-17$ years). Six patients underwent MRgLITT as a first-line treatment, 5 had undergone previous open surgery, and 3 had undergone oncological treatment that failed. The cases of $\mathrm{Pa}-$ tients 2 and 5 were reported previously. 6,25

Tumor histology revealed pilocytic astrocytoma $(n=6)$, ependymoma $(n=1)$, recurrent medulloblastoma $(n=1)$, choroid plexus xanthogranuloma $(n=2$ lesions in 1 patient), subependymal giant cell astrocytoma (SEGA; $n=1)$, and ganglioglioma $(n=1)$. Tumor locations were the frontal lobe $(\mathrm{n}=1)$, hypothalamus $(\mathrm{n}=1)$, thalamus $(\mathrm{n}=2)$, thalamus/ midbrain $(\mathrm{n}=1)$, ventricles $(\mathrm{n}=3)$, pons $(\mathrm{n}=1)$, and posterior fossa (vermian, peduncle, and tentorial; $n=3$ ). Framebased stereotaxis was used in 9 patients, and frameless stereotaxis was used in 2 patients.

\section{Surgical Procedure}

We previously described the surgical procedure used for these patients. ${ }^{6,25,34}$ However, our surgical technique has evolved with the incorporation of some technical details, including frameless stereotaxis for infratentorial tumors and use of intraoperative MRI. All MRgLITT procedures were performed using the Visualase thermal laser system (Medtronic). Laser trajectories were planned according to preoperative MRI (with and without contrast, diffusion tensor imaging, and functional MRI, when needed) and reexamined on the day of surgery using a Navigation image-guided surgery workstation (Stryker) or iPlan treatment-planning software (BrainLAB).

The choice of a frame-based (Leksell frame [Elekta $\mathrm{AB}]$ ) or frameless (VarioGuide [BrainLAB]) stereotactic technique was based on patient age, tumor size and location (supratentorial vs infratentorial), and tumor proximity to eloquent areas with lower tolerance for error (for which frame-based stereotaxis would be preferred). A biopsy sample was obtained during the same session for de novo lesions.

All procedures were performed after general anesthesia was induced. Our protocol included the administration of intravenous dexamethasone $(0.5 \mathrm{mg} / \mathrm{kg})$ at the beginning of the procedure and prophylactic antibiotics within half an hour of the skin incision. Prepping of the scalp entry zone was performed in a sterile fashion while avoiding clipping of the hair; the hair was parted and braided away from the surgical site to avoid hair pulling during drilling.

\section{Frame-Based Stereotaxis}

A frame-based stereotactic approach was preferred for small, deep, supratentorial tumors. The stereotactic frame and MR-lucent fiducial box were fitted in the operating theater. Once the patient was in the MRI suite, either a 1.5-T MRI (Signa EXCITE [GE Healthcare]) or intraop- erative 3-T MR-OR (Ingenia [Philips Medical Systems]) machine was used to acquire trajectory calculations. T2weighted Gd contrast-enhanced images were acquired as needed to visualize the tumor margins. All images were transferred to either the Stryker Navigation image-guided surgery workstation or the BrainLAB stereotactic iPlan software for coregistration of the acquired images to the Leksell frame coordinate system. Stereotactic trajectories were designed so that the laser could be inserted in the long axis of the tumor to allow for the maximal ablation diameter $(1.5 \mathrm{~cm})$ and multiple ablations along the same trajectory. Trajectories were reviewed on multiplanar 3D volume reconstructions of the MRI studies using the surgical view of the software and avoiding vascular or critical areas during laser insertion. Precise coordinates for the Leksell frame were used to perform the tumor biopsy and insertion of the cooling catheter and laser probe.

\section{Frameless Stereotaxis}

A frameless stereotactic approach was used primarily for infratentorial or superficial supratentorial tumors. Each patient was positioned prone with the head pinned (Sugita head holder [Mizuho]); the VarioGuide aiming device and MRI-guided navigation system were coregistered. Care was taken to leave enough distance between the VarioGuide and the scalp to allow for insertion of the Visualase cranial bolt.

\section{Technique}

A guided drill hole was made carefully in the skull to create a single trajectory in the bone; this is the single most important factor for avoiding trajectory error. A sedan needle biopsy kit was used to obtain tumor samples while taking care not to cause significant intratumoral bleeding that could affect the ablative properties of the tissue. The cranial bolt was screwed and secured to the bone with a stylet in place, passing through the Leksell frame or VarioGuide to maintain the same trajectory. Next, the cooling catheter and laser probe were inserted through the bolt to the calculated target; the depth was marked with sterile tape at the top of the cranial bolt and the laser insertion site..$^{6,25}$

\section{Laser Interstitial Thermal Therapy Procedure}

In the MRI suite, the laser was connected to the cooling pump, and the system software station was located in the reading room. A steady series of T1-weighted volume spoiled gradient-recalled MR images were obtained. A T1-weighted spin echo image including the laser probe was selected for the planning design in the thermal therapy software. ${ }^{5,34}$ The 1.5-T MRI machine was used to obtain MR thermal images in a fast radiofrequency spoiled gradient-recalled echo sequence (FOV $24 \times 24 \mathrm{~cm}$; matrix $256 \times 128$; echo time $20 \mathrm{msec}$; repetition time 45 msec; flip angle $30^{\circ}$; bandwidth $12.6 \mathrm{kHz}$ ). ${ }^{5}$ Later in our series, the intraoperative 3-T MR-OR machine was used to obtain images in a fast-field echo sequence (FOV $24 \times$ $24 \mathrm{~cm}$; matrix $252 \times 126$; echo time $10 \mathrm{msec}$; repetition time $40 \mathrm{msec}$; flip angle $19.50^{\circ}$; bandwidth $70 \mathrm{kHz}$ ). Acquisition of a single image required 6 seconds in the 1.5-T MRI machine and 5 seconds in the 3-T MR-OR machine. 


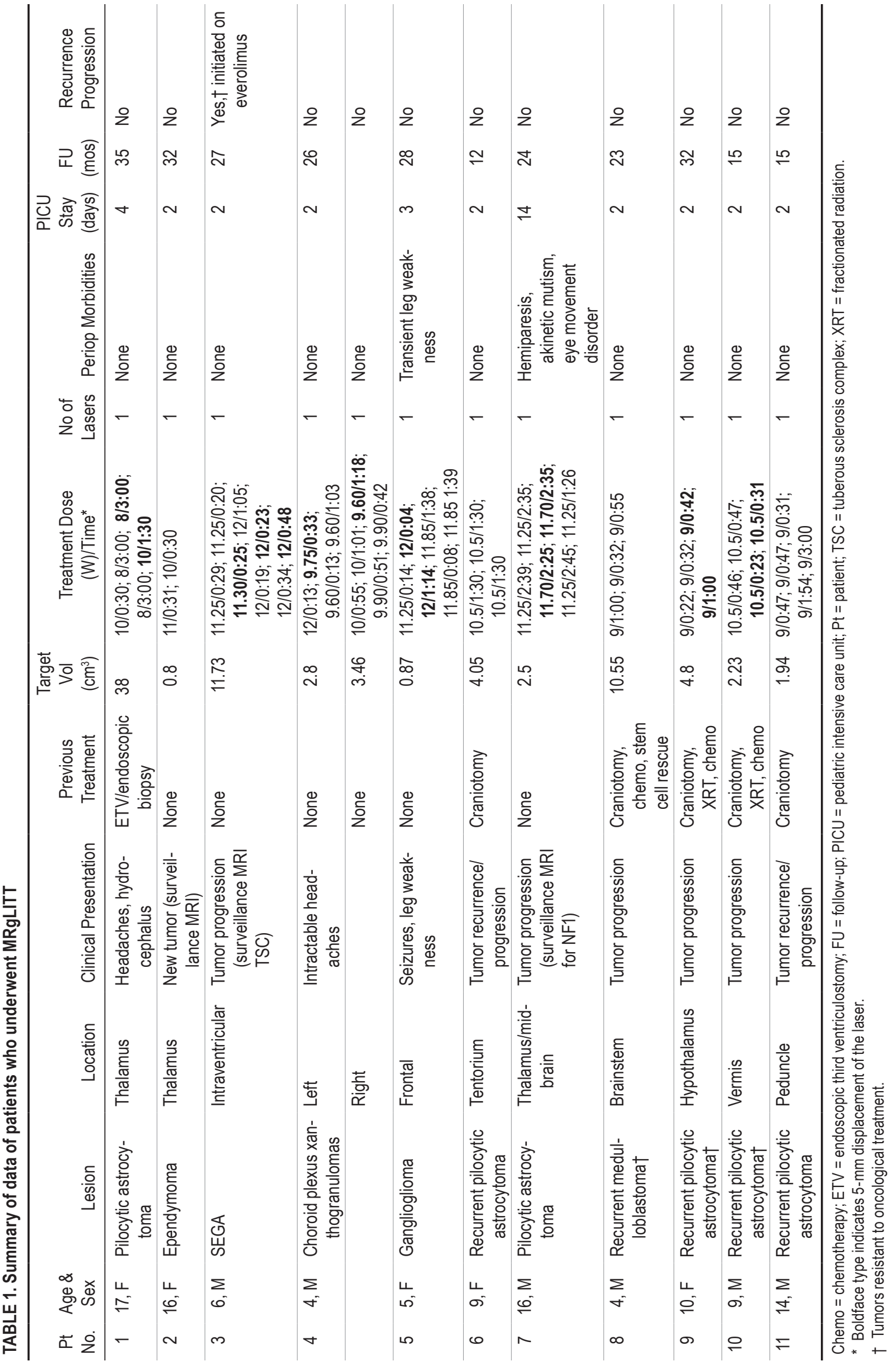


All MRgLITT procedures were performed using the Visualase thermal laser system, as we described previously. ${ }^{34}$ The thermal ablation plan was designed by localizing 1 crossed high-temperature limit in the area of desired maximal ablation and 3 crossed low-temperature limits to shield the interface with the normal brain. Preoperative diffusion tensor images were used to avoid damaging the vital tracts. A test dose using $25 \%$ of the laser power was applied to verify applicator position and proper synchronization of the images and laser software. Subsequently, treatment doses were applied (Table 1). Treatment was stopped by either the neurosurgeon, when the thermal map showed completed ablation (avoiding estimated tridimensional heating of the surrounding structures), or the software, when any temperature limit was reached. ${ }^{3}$ Additional thermal ablation zones were created, if needed, by repeating a second local dose to extend the area of ablation or by pulling the laser fiber inside the cooling catheter by $5 \mathrm{~mm}$. Initial and superimposed thermal ablation zones were saved to compose a final total ablation zone.

FLAIR and T1-weighted contrast-enhanced MR images were obtained postoperatively. Afterward, the patient was transported to the recovery room for removal of the frame (in the case of frame-based stereotaxis), cooling catheter, laser probe, and anchor. Each incision was closed with a single 3-0 Monocryl figure-8 stitch.

\section{Postoperative Regimen}

After the procedure, each patient was observed in the pediatric intensive care or step-down unit overnight with a plan to be discharged on postoperative Day 1. Dexamethasone was continued with a 2 -week weaning plan to prevent significant cerebral edema. A proton pump inhibitor was administered in conjunction with the dexamethasone for gastric protection. Routine postoperative visits occurred at 2 weeks and then every 2 to 3 months after treatment. Brain MRI was performed every 3 months (or earlier in the case of malignancy) to evaluate tumor size and volume.

\section{Data Analysis}

Post-ablation clinical outcomes, follow-up images, and times were recorded. Preoperative, intraoperative, and postoperative MR images were reviewed to calculate tumor volume in cubic centimeters by measuring axial, coronal, and sagittal views of the tumor in a T1-weighted contrastenhanced series of images. The number of observations and mean tumor volume $( \pm \mathrm{SD})$ at each time interval were calculated, and paired t-tests were performed to determine any statistical significance in tumor volume changes.

\section{Results \\ Patients and MRgLITT Data}

Surgical details are presented in Table 1. A single laser and multiple overlapping ablations were used in all the procedures. The mean laser dose was $10.23 \mathrm{~W}$, and the mean total ablation time was 68.95 seconds. Nine patients experienced no complications, whereas 2 experienced post-ablation morbidity: transient right leg weakness in 1 patient and transient hemiparesis, akinetic mutism, and eye movement disorder in the other. Both patients im- proved over time with rehabilitation to functional and independent levels. The mean hospital stay was 3.25 days; however, although 9 patients had a hospital stay of 2 days, Patients 1 and 7 had hospital stays of 4 and 14 days, respectively, as a result of cerebral edema. The mean followup time was 24.5 months.

\section{Volumetric Analyses}

The mean initial target volume was $6.79 \mathrm{~cm}^{3}$, and the mean immediate post-ablation volume was $7.86 \mathrm{~cm}^{3}$ (Fig. 1). The average tumor volume showed an expected slight increase immediately after the procedure ${ }^{23}$ that was not statistically significant. Tumor volume decreased in the first 3 months after surgery $(n=11 ; p=0.007)$ and continued to decrease by the 4- to 6-month follow-up $(\mathrm{n}=11$; mean volume $\left.2.61 \mathrm{~cm}^{3} ; \mathrm{p}=0.009\right)$. The average tumor volume increased slightly at the 7- to 9-month follow-up because of an outlier (Patient 3, who had an SEGA). Subsequent reductions in tumor size at the 7- to 9-, 10- to 12-, and 15- to 36-month follow-ups had no statistical significance. Mean and structural equation modeling were plotted at each time point (Fig. 2).

\section{Postoperative Imaging Evaluations}

Consistent postoperative imaging changes over time were observed in the ablated tumors. ${ }^{10}$ Figure 3 shows a summary of the cases in our series.

\section{Illustrative Cases \\ Case 1}

A 17-year-old girl presented with headaches and a calcified midbrain-thalamic pilocytic astrocytoma. This was the first case in our series in which MRgLITT was used, and it involved the longest follow-up time. Also, to date, this is the only reported case of cystic pilocytic astrocytoma treated with LITT. Figure 4A shows T1-weighted contrast-enhanced MR images at presentation for Patient 1. She was treated initially with an endoscopic third ventriculostomy, cyst fenestration, and biopsy (Fig. 4B) followed by MRgLITT (Fig. 4C). Postoperative MRI performed on the day of ablation confirmed the anticipated changes described by Hawasli et al., ${ }^{10}$ which consisted of a diffusion restriction ring at the margin of the treatment zone (Fig. 4D), a new ring of contrast enhancement, peritumoral ede$\mathrm{ma}$, and, in this case, an unexpected increase in lesion size (Fig. 4E). Peritumoral and cyst edema decreased after 2 weeks, and the new rim of enhancement decreased with time (Fig. 4F). The cyst was endoscopically refenestrated 6 months after surgery because of its increased size. The last follow-up at 36 months revealed no recurrence in this asymptomatic patient.

\section{Case 7}

A 16-year-old boy with neurofibromatosis Type 1 (NF1) and a growing symptomatic right midbrain-thalamic pilocytic astrocytoma was treated initially with MRgLITT (Fig. 5). This is the only case of this series in which clinical post-ablation edema was present. Preoperative tractography showed displacement of the fibers by the tumor (Fig. 


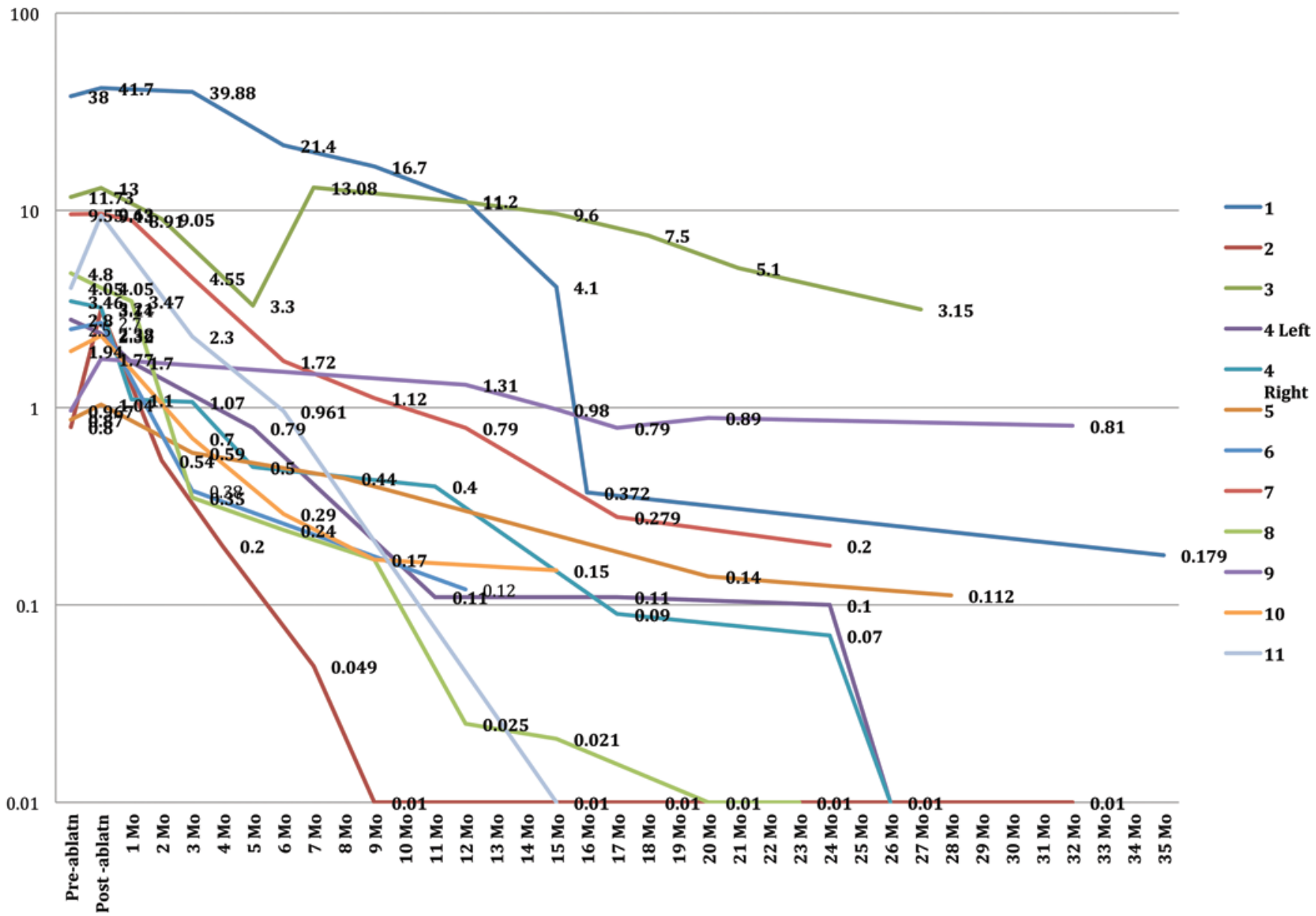

FIG. 1. Individual tumor volumes (in $\mathrm{cm}^{3}$ ) over time. ablatn = ablation.

5A). Ablation was achieved by means of superimposed treatments retracting the laser fiber inside the cooling catheter, and thermal mapping showed the estimated final damage (Fig. 5B). Postoperative FLAIR MR images were obtained on the day of ablation to display the treatment zone (Fig. 5C). The patient was experiencing postoperative somnolence and left-sided weakness, and CT without contrast performed after ablation revealed significant edema (Fig. 5D). One week after surgery, T1-weighted contrastenhanced MRI revealed a ring of contrast enhancement in the ablation zone and some resolution of the edema (Fig. $5 \mathrm{E})$. The enhancement rim progressively decreased with time (Fig. 5F).

\section{Case 8}

A 4-year-old boy with posterior fossa medulloblastoma and no metastatic disease underwent resection, chemotherapy, and stem cell rescue. After his second cycle of chemotherapy, a recurrent tumor was seen in the left pons (Fig. 6A). Stereotactic MRgLITT was offered as a palliative treatment. Preablation T2-weighted sagittal oblique MRI revealed the laser fiber inside the tumor (Fig. 6B). The ablation and final thermal damage estimate are shown in Fig. 6C. Postoperative MRI performed on the day of ablation showed typical new ring enhancement (Fig.
6D)..$^{10}$ Follow-up MRI revealed progressive reduction in ring-enhancement volume (Fig. $6 \mathrm{E}$ ). ${ }^{10}$ The patient did not receive any additional chemotherapy after the MRgLITT. The follow-up at 17 months found no recurrence. This is the first case in our series in which MRgLITT was applied as adjuvant therapy for a tumor that was resistant to treatment. At the time of this writing, the patient had returned to school with no deficits, and strict follow-up remained in place.

\section{Discussion}

During the past decade, LITT has been reported as an alternative to conventional surgical interventions for brain tumors, especially in adults with malignant or metastatic disease. . $^{3,12,22,36}$ However, reports of its use in pediatric cases are scarce. ${ }^{6}$

As a minimally invasive option, LITT offers several important advantages over other therapies for treatment of benign and malignant brain lesions. ${ }^{38}$ These advantages are particularly relevant to the pediatric population. For one, LITT has no limitations with respect to patient age or Karnofsky Performance Scale status. In addition, the procedure can be performed with the administration of local anesthesia, moderate sedation, or general anesthesia, ${ }^{12}$ and it is feasible and repeatable as often as indicated or as 


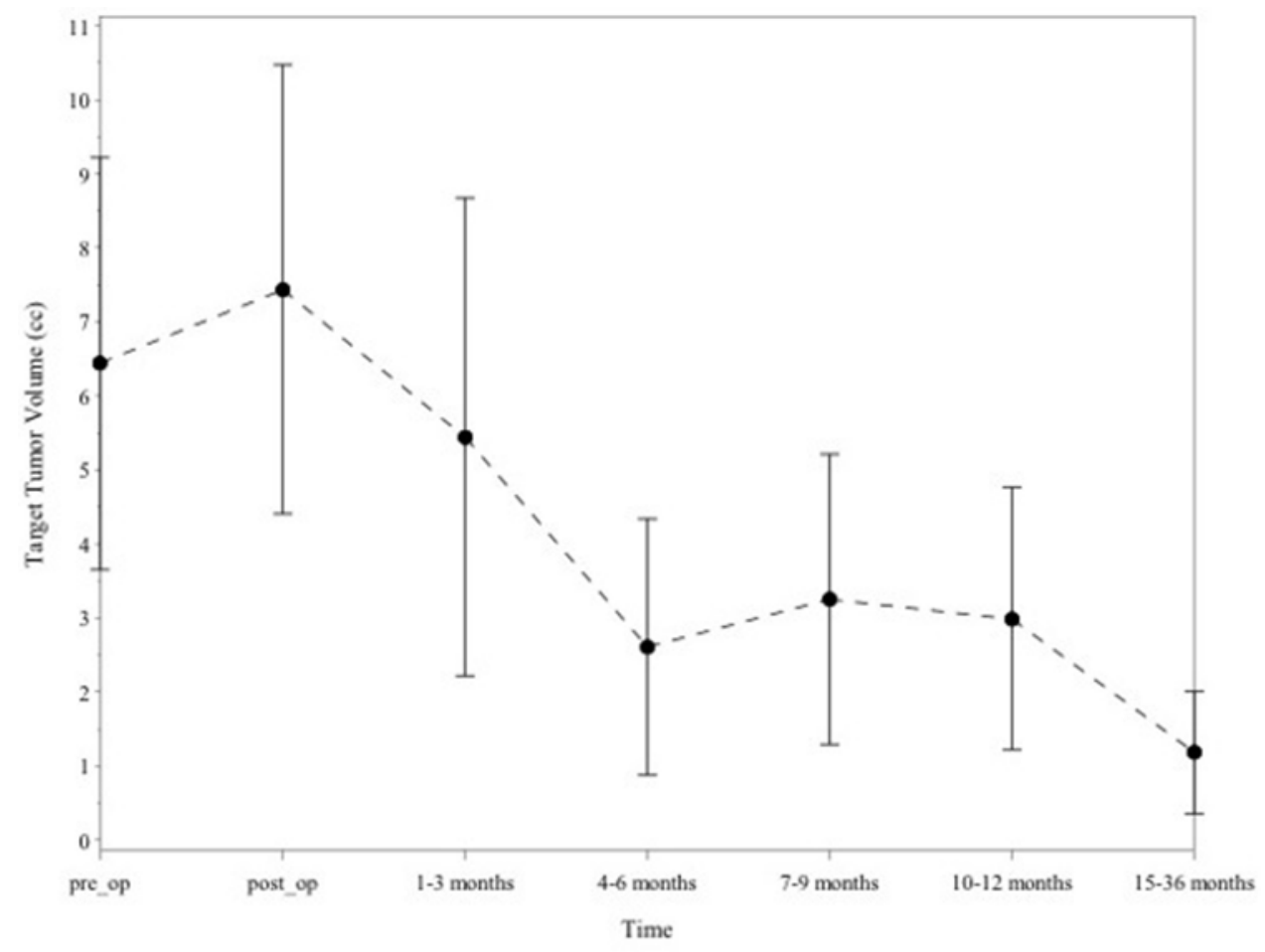

FIG. 2. Averaged tumor volumes (in $\mathrm{cm}^{3}$ ) over time.

soon as tumor recurrence occurs. Laser interstitial thermal therapy can be used as a de novo or subsequent treatment, alone or in conjunction with other therapies, because it has no dose limitations. It does not require ionizing radiation (which adds to its applicability in small children) and has no reported adverse effects apart from expected edema. The therapy is capable of producing a predictable injury pattern that conforms to the size and shape of the lesion(s). Laser interstitial thermal therapy can be achieved in a single surgical session, for 1 or multiple lesions, with 1 or multiple lasers, and with a single or added laser ablation for each lesion. ${ }^{38}$ Furthermore, LITT is MRI compatible, reduces operating time (especially in the setting of intraoperative MRI), and shortens the hospital stay. Finally, results of the procedure are cosmetically pleasing, it does not require suture removal, and it generates minimal discomfort, which provides patients and their families with an overall less traumatic experience.

Reported preclinical studies ${ }^{15,18,19}$ have shown that LITT delivers controlled, customizable, precise, real-time thermal ablation under MR guidance. ${ }^{34}$ Laser interstitial thermal therapy uses light absorption to induce thermocoagulation of target brain tissue. Chromospheres in the tumor absorb the photons emitted by the optical fiber, releasing heat and leading to cell death by direct thermal injury or subsequent apoptosis. ${ }^{11,29}$ The time/temperature ratio induced by the laser applicator is critical and has to be controlled. A fast or significant increase in temperature may cause explosive carbonization or vaporization of the target tissue, referred to as a "popcorn effect." 27 Nonetheless, the clinical indications of LITT are dynamic and innovative in consonance with neurosurgeon expertise with the application and limitations of the technology.
Here, we present our initial experience with MRgLITT for 4 supratentorial, 3 intraventricular, 1 thalamic/midbrain, 1 brainstem, and 3 infratentorial pediatric tumors. These cases represent select patients with deep-seated brain tumors, recurrent tumors, and other tumors that are difficult to identify intraoperatively (Case 5). Nine patients received MRgLITT as the initial therapy, whereas the other 3 patients (Cases 8-10) were offered MRgLITT as an alternative treatment for progressive disease when the tumor became resistant to adjuvant therapies. Volumetric followup, as noted in Fig. 1, showed a progressive cytoreductive tumor effect. In addition, in 3 patients whose tumors were refractory to chemotherapy, the Karnofsky Performance Scale $^{15}$ status improved from 70 to 90 after MRgLITT, and chemotherapy had been discontinued since the ablation. At the time of this writing, these patients were still being kept under close oncological watch. Although this is a small cohort of tumors with a short follow-up, longer follow-up and later reports will further evaluate whether the effect of the laser is sufficient to prevent recurrence and whether a second ablation and/or different adjuvant treatment will be required.

We used a single laser trajectory in each patient, and no repeated treatments were required. However, in retrospect, based on the premise that the laser treatment creates elliptical lesions approximately $1.5-2 \mathrm{~cm}$ along the fiber and $1.0-1.5 \mathrm{~cm}$ transverse to the fiber, ${ }^{42}$ the outlier patient with an SEGA (Case 3 [Fig. 3]) required the use of 2 laser probes given the tumor's large size and shape. The initial ablation destroyed only part of the tumor; a second trajectory should have aimed to ablate the tumor at the subependymal layer to detach it from its origin and vascularity. However, this patient was treated with everolimus begin- 

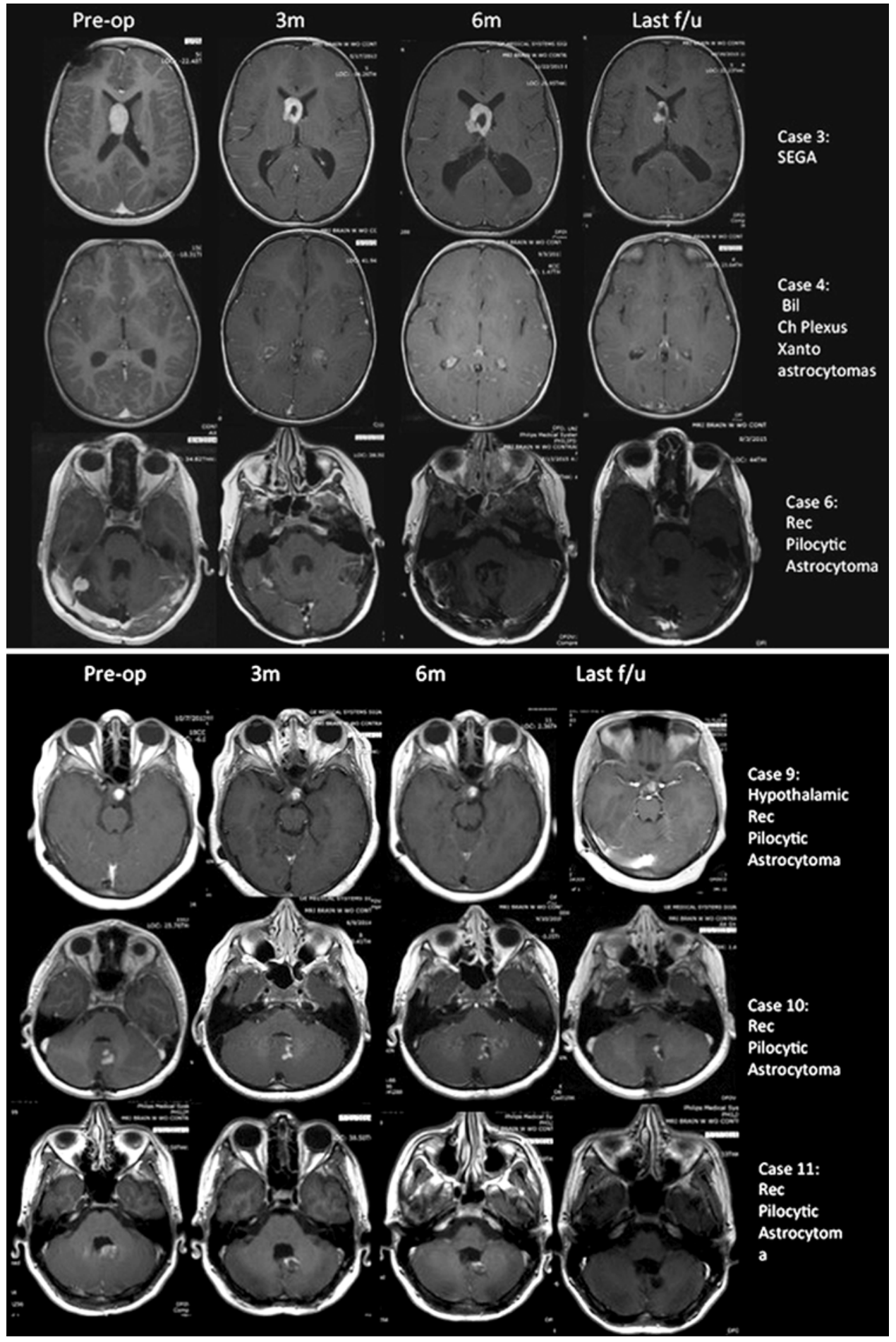

FIG. 3. Synopsis of tumor cases and MRI changes over time. The cases of Patients 2 and 5 have been reported previously. ${ }^{6,25}$ Bil Ch Plexus Xanto = bilateral choroid plexus xanthogranulomas; $f / u=$ follow-up; $m=$ months; Rec. $=$ recurrent.

ning 7 months after ablation, and the tumor has since further decreased in size. Therefore, coadjuvant therapy has been more effective in this case.

The total hospital stay in this series was shorter than that in previous studies. ${ }^{10,12}$ Nine of the 11 patients had an overall stay of 2 days, but 2 patients with deep lesions (Patients 1 and 7) stayed longer as a result of post-ablation edema.
Overcaution also was a factor in Patient 1 , who had a cystic thalamic pilocytic astrocytoma. It is interesting to note that in this case, the already fenestrated cystic part of the tumor increased in size after ablation, probably because of the proteinaceous content of the cyst. We followed the cyst for 6 months, and although it decreased in size over time, we considered it appropriate to re-fenestrate it en- 

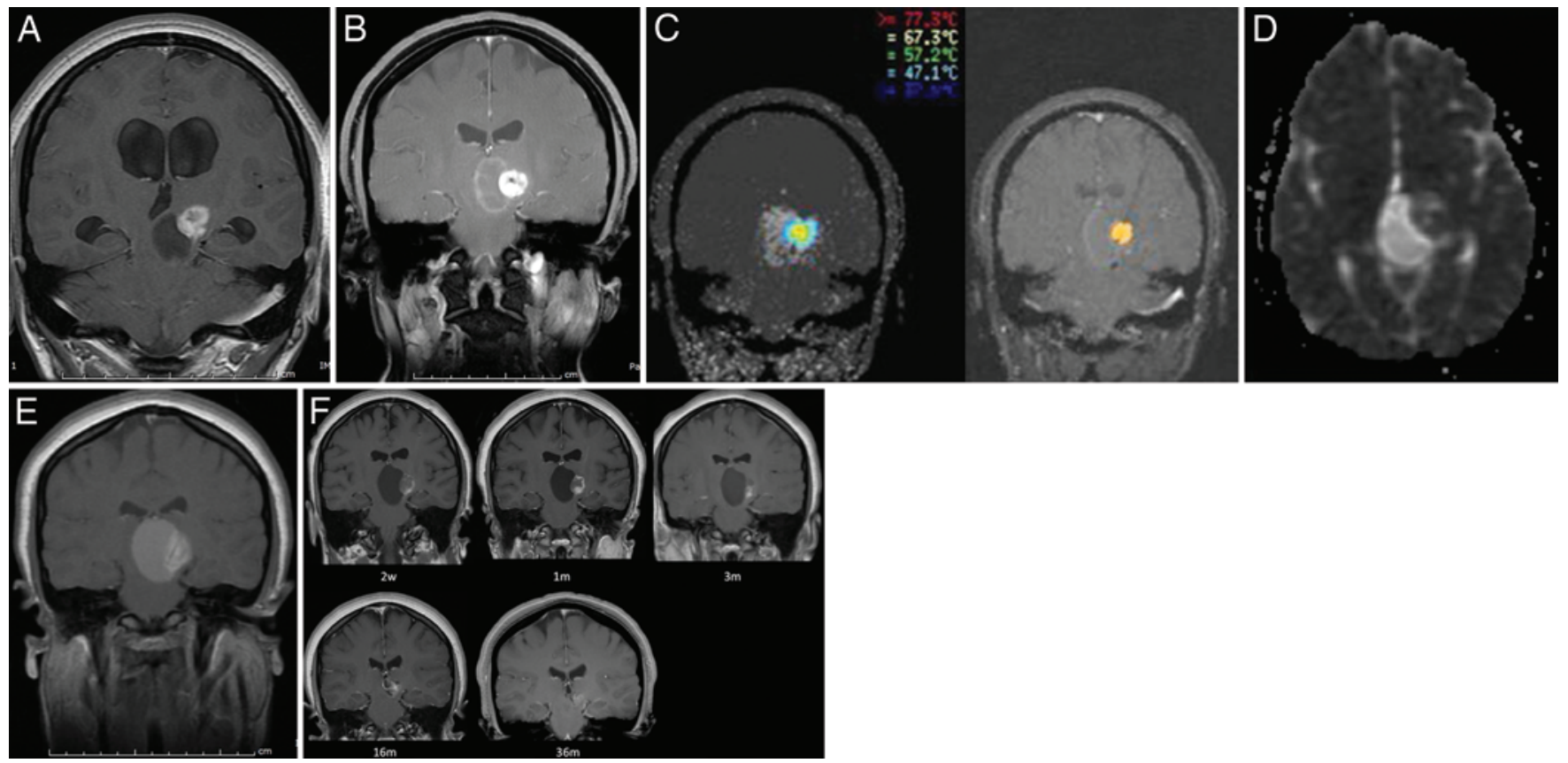

FIG. 4. Case 1. A: Initial MR image showing a left subthalamic/thalamic pilocytic astrocytoma with a cystic component measuring $29 \times 23 \times 23 \mathrm{~mm}$ and an associated heterogeneous solid component measuring $17 \times 17 \times 15 \mathrm{~mm}$. The tumor caused obstructive hydrocephalus. The patient was treated initially with an endoscopic third ventriculostomy, cyst fenestration, and biopsy. B: Six months after initial endoscopic intervention, preablation T1-weighted MRI with Gd enhancement showing a solid-component left subthalamic/thalamic lesion measuring $20 \times 18 \mathrm{~mm}$. C: Intraoperative images of LITT thermal map and irreversible damage estimate. D: Postoperative MR image on the day of ablation showing a ring of diffusion restriction at the edge of the ablated tumor. E: Post-ablation T1-weighted contrast-enhanced MR image showing enhancement in the ablated area, peritumoral edema, and an interestingly increased cyst size. F: Follow-up T1-weighted MR images with contrast at 2 weeks and at 1, 3, 16, and 36 months. The patient had repeat endoscopic cyst fenestration 6 months after ablation.

doscopically. This patient had the longest follow-up time in this series, and the residual part of the tumor seen on MRI corresponded to the calcified part of the lesion. Nonetheless, at the time of this writing, the patient was clinically asymptomatic and fully functional with no tumor regrowth after 3 years. Patient 7, who had NF1 and a midbrain-thalamic pilocytic astrocytoma, experienced severe acute post-ablation edema that caused dense hemiparesis, akinetic mutism, and eye movement disorder. We hypothesize that overheating of the 2D target area and the longer ablation time contributed to conductive heating of the peritumoral tissue. The patient showed concurrent hyponatremia and intraoperative fluid overload. Since then, we have been carefully monitoring these parameters intraoperatively. This patient was managed with higher doses of steroids ( $1 \mathrm{mg} / \mathrm{kg} /$ day) and $3 \% \mathrm{NaCl}$. The steroids were tapered off 2 weeks after surgery. The patient experienced progressive improvement after rehabilitation and, 18 months after ablation, had only residual mild hand weakness and dysarthria.

Patient 5 presented with seizures and postictal leg weakness. Seizure evaluation showed localization-related epilepsy caused by a frontal lesion believed to be a hamartoma. The patient experienced improvement in her leg weakness and became seizure free after ablation. Similar to other researchers, ${ }^{7,40}$ we have found that LITT is effective for select patients with focal epilepsy. ${ }^{34}$

Post-ablation MRI features have been widely described ${ }^{10}$ and were observed in our study. Initially, the tumor exhibits hemorrhagic necrosis, a new ring of diffusion restriction and contrast enhancement delineating the ablation zone, and peritumoral edema. Our follow-up images showed a lack of diffusion restriction, progressive reduction in ringenhancement size, and resolution of edema. In addition, volumetric outcomes were consistent; in only 1 case of regrowth (SEGA) was ablation considered suboptimal.

\section{Limitations}

We previously reported our experience with the Visualase thermal laser system..$^{6,25,34}$ The system was designed to simultaneously control ablation and generate color-coded temperature maps, which are overlaid on MR images. . $^{13,22,34}$ An irreversible damage zone is calculated based on time and temperature data from individual voxels in the treatment area $^{18}$ and subsequent cellular death. ${ }^{39}$ To date, based on this initial experience, we do not have enough data to specify the different time/temperature ratios that are adequate for the various types of tissue and brain tumors. We realize that a particular set of parameters or thresholds may provide a better prediction of ablation for each type of tissue and would demonstrate how the laser should be used for diverse pathologies. However, some studies have found that tissue damage caused by thermal ablation can be tissue-type dependent. ${ }^{42}$ Moreover, tumors with similar histological grades have been shown to have variable responses to LITT, ${ }^{16,30-32}$ which makes it very challenging to standardize tumor-specific laser protocols. 

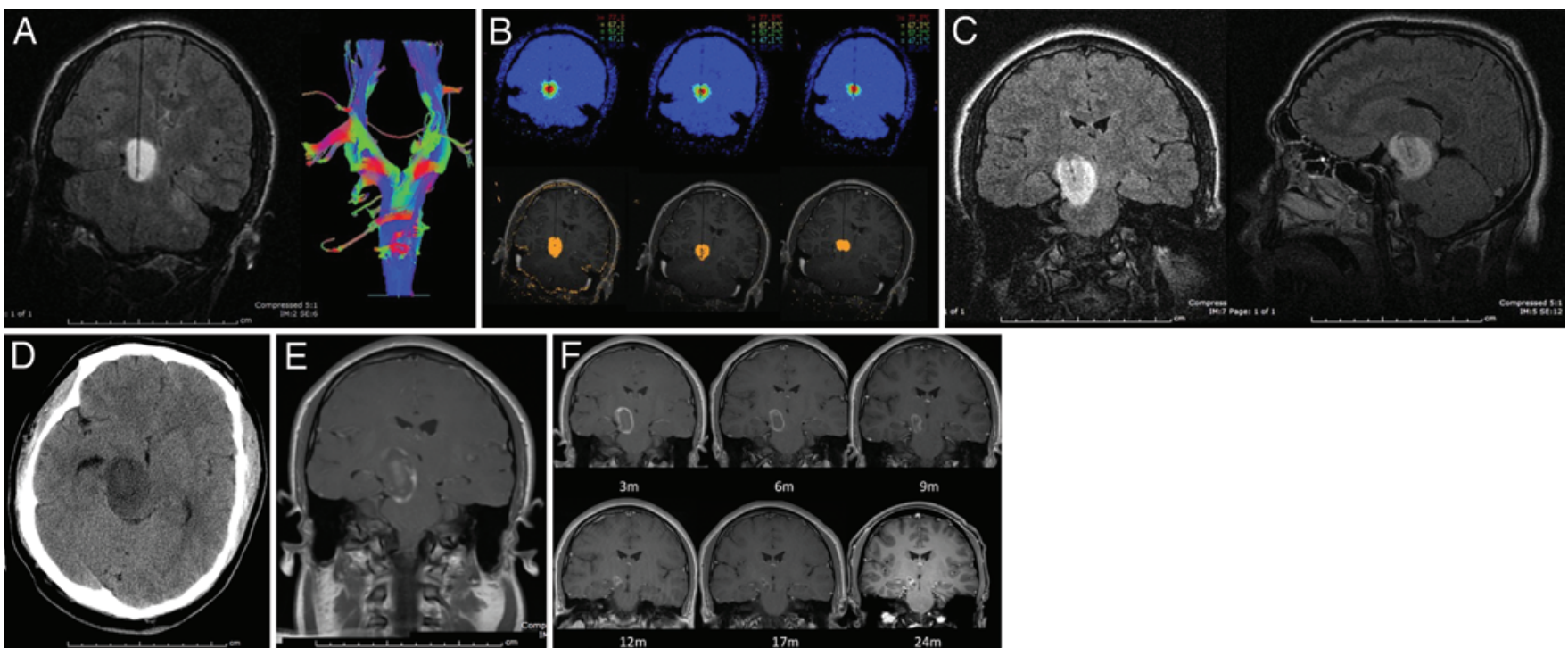

FIG. 5. Case 7. A: Pre-ablation coronal T2-weighted MR and tractography images showing laser placement and tractography in a 16-year-old patient with NF1 and a growing symptomatic right midbrain/thalamic pilocytic astrocytoma treated with de novo LITT. B: LITT-added ablations and final damage estimation. C: Post-ablation coronal and sagittal T2-weighted FLAIR MR images showing the ablation zone and restricted edema. D: Noncontrast CT scan acquired after ablation revealing significant edema. E: Post-Gd T1-weighted MR image 1 week after ablation showing the ablation ring of contrast enhancement with some resolution of the edema. F: Follow-up coronal T1-weighted contrast-enhanced MR images obtained 3, 6, 9, 12, 17, and 24 months after ablation showing reduced tumor volume.

As explained earlier, the software links the temperature limits and the voxels in the image. The laser is turned off automatically if any temperature limit is reached. ${ }^{7,34} \mathrm{Nev}-$ ertheless, the current software is restricted to using single or 2D T1-weighted spin echo sequences. The selected stat- ic image used for target planning and mapping includes the applicator and the visible portion of the tumor. Consequently, there is a lack of tumor volumetric data, which forces the surgeon to calculate the area of thermal damage on the different planes on pre-ablation MR images.
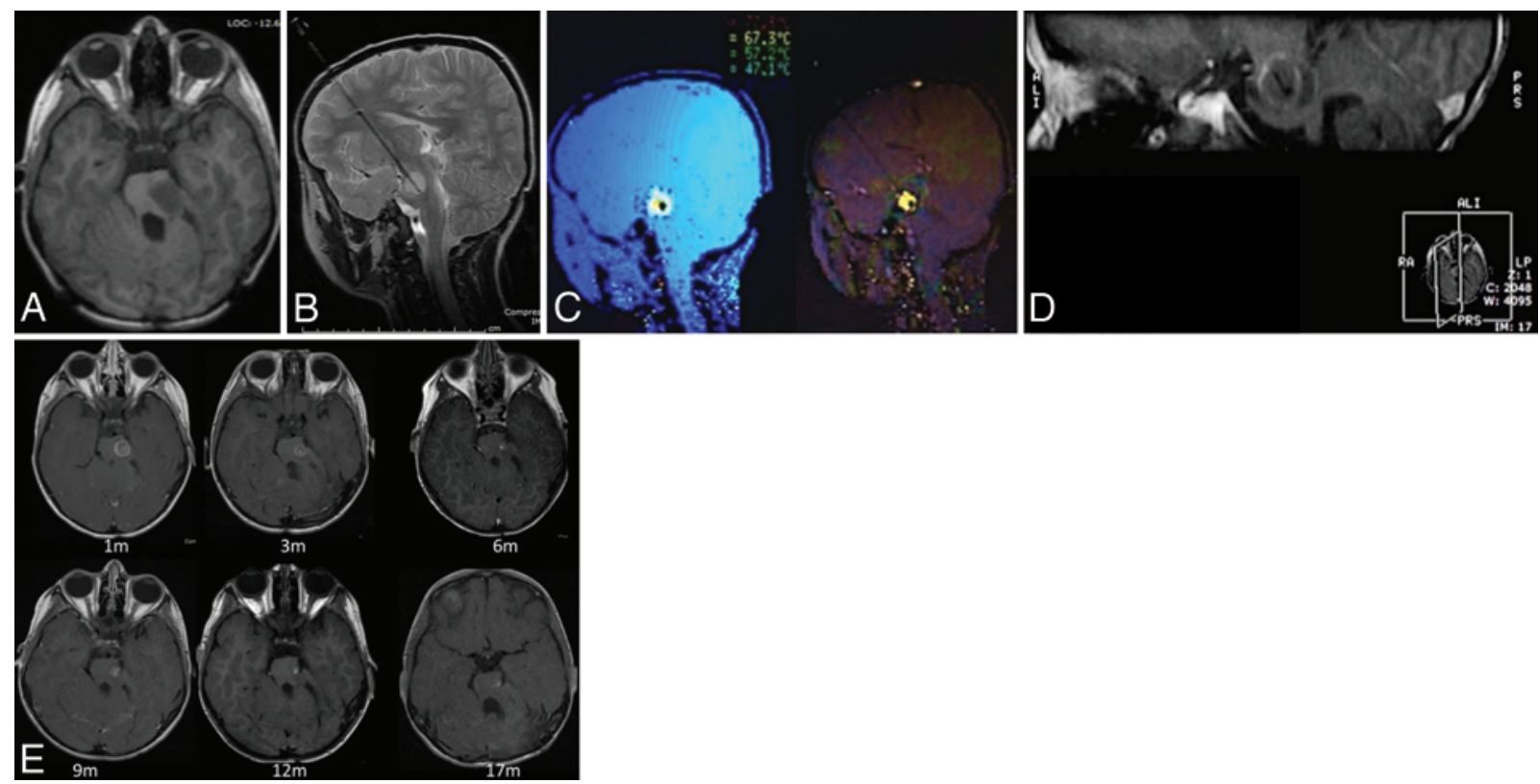

FIG. 6. Case 8. Images obtained in a 4-year-old boy with no metastatic disease showing a recurrent medulloblastoma that was resistant to chemotherapy. A: After the second cycle of chemotherapy, a recurrent tumor is seen in the left pons. B: Pre-ablation T2-weighted sagittal oblique MR image showing laser location inside the medulloblastoma. C: LITT-added ablation and final damage estimation. D: Postoperative sagittal contrast-enhanced MR image with contrast on the day of ablation demonstrating the new, thin uniform contrast-enhanced ring in the treatment zone. E: Follow-up axial T1-weighted contrast-enhanced MR images at $1,3,6,9,12$, and 17 months showing evolution of the ablated tumor. 
Furthermore, the cross-marked symbols used for the thermal limits are not exact enough to delineate the border between the lesion and healthy surrounding fibers. This factor is particularly critical when precise microsurgical limits of ablation are crucial for preventing postprocedure morbidity. In our series, the estimated sharp transition between the target ablation area and viable tissue ${ }^{35}$ looked to be adequate for the thermal damage image. However, the extended injury from the laser can be larger. Because the ablation zone depends on applied laser power, if the heat produced is maintained above a threshold temperature (usually $45^{\circ} \mathrm{C}$ ), the tissue will irreversibly coagulate and change the optical properties of energy absorption, which would lead to a localized increase in tumor temperature and a spread of thermal energy to the neighboring regions. Hence, while the lesion surface is being ablated, adjacent tissue may become inadvertently coagulated and show post-ablation apoptosis and edema.

When reviewing the post-ablation T2-weighted FLAIR MR images of the patient with a brainstem tumor and postoperative edema (Fig. 5C), we can appreciate the signal outside the area of ablation. Because ablation was performed in the coronal plane, leakage of thermal energy to the surrounding tissue in the axial and sagittal planes probably contributed to the significant post-ablation swelling. Using the current software, we might avoid this leakage by setting the temperature limits inside the borders of the lesion and by taking care not to extend the ablation further once the planned thermal map has been reached.

The results of preliminary quantitative analysis of our outcomes, which reveal progressive reduction in tumor volume, are promising. However, this was a small heterogeneous series of patients with short- to medium-length follow-ups.

\section{Future Considerations}

We have discussed the current indications for MRgLITT. Our results suggest that MRgLITT can be considered a de novo treatment or an alternative to second-look procedures for surgically challenging recurrent or residual tumors. Furthermore, because the blood-brain barrier is modulated or opened during, and for a defined period after, MRgLITT, the effect of targeted therapy might be improved in the case of malignancy. ${ }^{37,38}$ The addition of MRgLITT to nanotechnology may be of great value to nanoparticle-enhanced ablation treatments and enable much deeper optical penetration into the tumor.,20,24

Magnetic resonance-guided LITT systems are expected to evolve and be compatible with robotic and computerized simulation applications. Upgrades should include improved tools for planning and insertion, volumetric MR-guided ablation monitoring, and tuning optimal temperature limits. ${ }^{29}$

\section{Conclusions}

Surgical and technological innovations are necessary for the advancement of neurosurgery. As with any new technology, there is a learning curve associated with MRgLITT that requires a full understanding of laser properties and the optical characteristics of different tissues.
We have reported the initial outcomes of MRgLITT in a heterogeneous sample of pediatric intracranial tumors. This technique has proven to be an effective first- or second-line treatment for select patients.

Laser interstitial thermal therapy offers many advantages that could positively influence compliance to treatment and improve quality of life for pediatric patients with intracranial neoplasms by avoiding or attenuating the collateral effects of adjuvant therapies. Nonetheless, this is only an early statement, and multiinstitutional clinical trials are necessary to evaluate the use of LITT in different types of tumors to standardize practices and create treatment algorithms.

\section{Acknowledgments}

We thank Sean Huckins, MS, for his contribution in planning the cases. We also thank the Radiology and Operating Room teams at SUNY Upstate Medical University for their enthusiasm in making this research possible.

\section{References}

1. Baek SK, Makkouk AR, Krasieva T, Sun CH, Madsen SJ, Hirschberg H: Photothermal treatment of glioma; an in vitro study of macrophage-mediated delivery of gold nanoshells. J Neurooncol 104:439-448, 2011

2. Bettag M, Ulrich F, Schober R, Fürst G, Langen KJ, Sabel M, et al: Stereotactic laser therapy in cerebral gliomas. Acta Neurochir Suppl (Wien) 52:81-83, 1991

3. Carpentier A, Chauvet D, Reina V, Beccaria K, Leclerq D, McNichols RJ, et al: MR-guided laser-induced thermal therapy (LITT) for recurrent glioblastomas. Lasers Surg Med 44:361-368, 2012

4. Carpentier A, McNichols RJ, Stafford RJ, Guichard JP, Reizine D, Delaloge S, et al: Laser thermal therapy: real-time MRI-guided and computer-controlled procedures for metastatic brain tumors. Lasers Surg Med 43:943-950, 2011

5. Carpentier A, McNichols RJ, Stafford RJ, Itzcovitz J, Guichard JP, Reizine D, et al: Real-time magnetic resonance-guided laser thermal therapy for focal metastatic brain tumors. Neurosurgery 63 (1 Suppl 1):ONS21-ONS29, 2008

6. Choi H, Tovar-Spinoza S: MRI-guided laser interstitial thermal therapy of intracranial tumors and epilepsy: State-ofthe-art review and a case study from pediatrics. Photonics Lasers Med 3:107-115, 2014

7. Curry DJ, Gowda A, McNichols RJ, Wilfong AA: MR-guided stereotactic laser ablation of epileptogenic foci in children. Epilepsy Behav 24:408-414, 2012

8. Earle KM, Carpenter S, Roessmann U, Ross MA, Hayes JR, Zeitler E: Central nervous system effects of laser radiation. Fed Proc 24 (Suppl 14):129, 1965

9. Fine S, Klein E, Nowak W, Scott RE, Laor Y, Simpson L, et al: Interaction of laser radiation with biologic systems. I. Studies on interaction with tissues. Fed Proc 24 (Suppl 14):35-47, 1965

10. Hawasli AH, Bagade S, Shimony JS, Miller-Thomas M, Leuthardt EC: Magnetic resonance imaging-guided focused laser interstitial thermal therapy for intracranial lesions: singleinstitution series. Neurosurgery 73:1007-1017, 2013

11. Heisterkamp J, van Hillegersberg R, Zondervan PE, IJzermans JN: Metabolic activity and DNA integrity in human hepatic metastases after interstitial laser coagulation (ILC). Lasers Surg Med 28:80-86, 2001

12. Jethwa PR, Barrese JC, Gowda A, Shetty A, Danish SF: Magnetic resonance thermometry-guided laser-induced thermal therapy for intracranial neoplasms: initial experience. Neurosurgery 71 (1 Suppl Operative):133-144, 144-145, 2012 
13. Jethwa PR, Lee JH, Assina R, Keller IA, Danish SF: Treatment of a supratentorial primitive neuroectodermal tumor using magnetic resonance-guided laser-induced thermal therapy. J Neurosurg Pediatr 8:468-475, 2011

14. Kangasniemi M, McNichols RJ, Bankson JA, Gowda A, Price RE, Hazle JD: Thermal therapy of canine cerebral tumors using a $980 \mathrm{~nm}$ diode laser with MR temperature-sensitive imaging feedback. Lasers Surg Med 35:41-50, 2004

15. Karnofsky DA, Burchenal JH: The clinical evaluation of chemotherapeutic agents in cancer, in Macleod CM (ed): Evaluation of Chemotherapeutic Agents. New York: Columbia University Press, 1949, pp 191-205

16. Leonardi MA, Lumenta CB, Gumprecht HK, von Einsiedel GH, Wilhelm T: Stereotactic guided laser-induced interstitial thermotherapy (SLITT) in gliomas with intraoperative morphologic monitoring in an open MR-unit. Minim Invasive Neurosurg 44:37-42, 2001

17. Marchesini R, Andreola S, Emanuelli H, Melloni E, Schiroli A, Spinelli P, et al: Temperature rise in biological tissue during Nd:YAG laser irradiation. Lasers Surg Med 5:75-82, 1985

18. McNichols RJ, Gowda A, Kangasniemi M, Bankson JA, Price RE, Hazle JD: MR thermometry-based feedback control of laser interstitial thermal therapy at $980 \mathrm{~nm}$. Lasers Surg Med 34:48-55, 2004

19. McNichols RJ, Kangasniemi M, Gowda A, Bankson JA, Price RE, Hazle JD: Technical developments for cerebral thermal treatment: water-cooled diffusing laser fibre tips and temperature-sensitive MRI using intersecting image planes. Int J Hyperthermia 20:45-56, 2004

20. Missios S, Bekelis K, Barnett GH: Renaissance of laser interstitial thermal ablation. Neurosurg Focus 38(3):E13, 2015

21. Mohammadi AM, Hawasli AH, Rodriguez A, Schroeder JL, Laxton AW, Elson P, et al: The role of laser interstitial thermal therapy in enhancing progression-free survival of difficult-to-access high-grade gliomas: a multicenter study. Cancer Med 3:971-979, 2014

22. Norred SE, Johnson JA: Magnetic resonance-guided laser induced thermal therapy for glioblastoma multiforme: a review. BioMed Res Int 2014:761312, 2014

23. Patel N, Jethwa P, Barrese J, Hargreaves E, Danish S: Volumetric trends associated with MRI-guided laser-induced thermal therapy (LITT) for intracranial tumors. Lasers Surg Med Aug 45:362-369, 2013

24. Rahmathulla G, Recinos PF, Kamian K, Mohammadi AM, Ahluwalia MS, Barnett GH: MRI-guided laser interstitial thermal therapy in neuro-oncology: a review of its current clinical applications. Oncology 87:67-82, 2014

25. Riordan M, Tovar-Spinoza Z: Laser induced thermal therapy (LITT) for pediatric brain tumors: case-based review. Transl Pediatr 3:229-235, 2014

26. Schulze PC, Vitzthum HE, Goldammer A, Schneider JP, Schober R: Laser-induced thermotherapy of neoplastic lesions in the brain - underlying tissue alterations, MRI-monitoring and clinical applicability. Acta Neurochir (Wien) 146:803-812, 2004

27. Schwarzmaier H, Eickmeyer F, Fiedler V, Ulrich F: Basic principles of laser induced interstitial thermotherapy in brain tumors. Med Laser Appl 17:147-158, 2002

28. Sloan AE, Ahluwalia MS, Valerio-Pascua J, Manjila S, Torchia MG, Jones SE, et al: Results of the NeuroBlate System first-in-humans Phase I clinical trial for recurrent glioblastoma: clinical article. J Neurosurg 118:1202-1219, 2013

29. Stafford RJ, Fuentes D, Elliott AA, Weinberg JS, Ahrar K: Laser-induced thermal therapy for tumor ablation. Crit Rev Biomed Eng 38:79-100, 2010

30. Svaasand LO, Boerslid T, Oeveraasen M: Thermal and opti- cal properties of living tissue: application to laser-induced hyperthermia. Lasers Surg Med 5:589-602, 1985

31. Svaasand LO, Ellingsen R: Optical penetration in human intracranial tumors. Photochem Photobiol 41:73-76, 1985

32. Svaasand LO, Ellingsen R: Optical properties of human brain. Photochem Photobiol 38:293-299, 1983

33. Torres-Reveron J, Tomasiewicz HC, Shetty A, Amankulor NM, Chiang VL: Stereotactic laser induced thermotherapy (LITT): a novel treatment for brain lesions regrowing after radiosurgery. J Neurooncol 113:495-503, 2013

34. Tovar-Spinoza Z, Carter D, Ferrone D, Eksioglu Y, Huckins $\mathrm{S}$ : The use of MRI-guided laser-induced thermal ablation for epilepsy. Childs Nerv Syst 29:2089-2094, 2013

35. Tracz RA, Wyman DR, Little PB, Towner RA, Stewart WA, Schatz SW, et al: Comparison of magnetic resonance images and the histopathological findings of lesions induced by interstitial laser photocoagulation in the brain. Lasers Surg Med 13:45-54, 1993

36. Voigt J, Torchia M: Laser interstitial thermal therapy with and without MRI guidance for treatment of brain neoplasms. A systematic review of the literature. Photonics Lasers Med 3:77-93, 2014

37. von Tempelhoff W, Toktamis S, Schwarzmaier H, Eickmeyer F, Niehoff H, Ulrich F: LITT (laser-induced interstitial thermotherapy) of benign and malignant gliomas in the open MRT (0.5 Tesla, GE Signa SP). Med Laser Appl 17:170-178, 2002

38. von Tempelhoff W, Ulrich F: Laser interstitial thermal therapy (LITT) of the brain. Photonics Lasers Med 3:67-69, 2014

39. Welch AJ, van Gemert MJC: Optical-Thermal Response of Laser-Irradiated Tissue, ed 1. New York: Springer, 1995

40. Willie JT, Laxpati NG, Drane DL, Gowda A, Appin C, Hao C, et al: Real-time magnetic resonance-guided stereotactic laser amygdalohippocampotomy for mesial temporal lobe epilepsy. Neurosurgery 74:569-585, 2014

41. Yaroslavsky AN, Schulze PC, Yaroslavsky IV, Schober R, Ulrich F, Schwarzmaier HJ: Optical properties of selected native and coagulated human brain tissues in vitro in the visible and near infrared spectral range. Phys Med Biol 47:2059-2073, 2002

42. Yung JP, Shetty A, Elliott A, Weinberg JS, McNichols RJ, Gowda A, et al: Quantitative comparison of thermal dose models in normal canine brain. Med Phys 37:5313-5321, 2010

\section{Disclosures}

The authors report no conflict of interest concerning the materials or methods used in this study or the findings specified in this paper.

\section{Author Contributions}

Conception and design: both authors. Acquisition of data: TovarSpinoza. Analysis and interpretation of data: both authors. Drafting the article: Tovar-Spinoza. Critically revising the article: Tovar-Spinoza. Reviewed submitted version of manuscript: Tovar-Spinoza. Approved the final version of the manuscript on behalf of both authors: Tovar-Spinoza. Statistical analysis: Tovar-Spinoza. Administrative/technical/material support: TovarSpinoza. Study supervision: Tovar-Spinoza.

\section{Correspondence}

Zulma Tovar-Spinoza, Department of Neurosurgery, SUNY Upstate Medical University, 604 Jacobsen Hall, 750 E. Adams St., Syracuse, NY 13210. email: tovarspz@upstate.edu. 